O'Brien, C. P., Volpicell, L. A. \& Volpiceld, J. R. (1996) Naltrexone in the treatment of alcoholism: a clinical review. Alcohol, 13, 35-39.

POLCH, J. M., ARMOR, D. \& BRAIKER, H. (1981) The Course of Alcoholism Four Years After Treatment. New York: John Wiley and Sons.

POUlsen, E. H., Loft, S., ANDERSEN, J. R., et al (1992) Disulfiram therapy adverse drug reaction and interactions. Acta Psychiatrica Scandinavida, 86, 59-66.

Shaw, S. (1985) The disease concept of dependence. In The Misuse of Alcohol: Crucial Issues on Dependence. Treatment and Prevention (eds N. Heather, I. Robertson \& P. Davies). London: Croom Helm.

SUNDERMAN, F. W. (1958) Dithiocarb: a new therapeutic agent for persons exposed to nickel carbonyl. American Journal of Medical Science, 236, 26-30.
VOlPICELU, J. R., RHINES, K. C., RHINES, J. S., et al (1997) Naltrexone and alcohol dependence. Archives of General Psychiatry, 64, 1737-1742.

WORID DRINK TRENDS (1992) World Drink Thends, International Beverage Alcohol Consumption and Production Trends. Henley-on-Thames: Produktschap voor Gedistillerde Dranken and NTC Publications.

* Jonathan Chick, Senior Lecturer in Psychiatry, University of Edinburgh, Royal Edinburgh Hospital, Edinburgh EHIO 5HF; and Colin Brewer, Medical Director, The Stapleford Centre, London SWI 9PN

*Correspondence

\title{
Use of high-dose depot neuroleptics
}

\author{
N. Purandare, L. Aitken, P. Joshi and C. S. Thomas
}

Aims and methods To identify and reduce the number of patients recelving depot neuroleptics above the British National Formulary maximum. The medical records were scrutinised and individual consultants were informed of the results.

Results For the team involved in this audit, there was a significant reduction in the prescription of high-dose depot medication, but this did not generalise to other teams.

Clinical implications Different teams should repeat the audit and a new depot card has been developed.

The use of high doses of neuroleptics is being increasingly questioned both on the grounds of safety and efficacy (King, 1994). The occasional association of sudden death in psychiatric patients and high-dose antipsychotic treatment has prompted comment and a consensus statement (Hirsch \& Barnes, 1994; Thompson, 1994). Side-effects such as sedation, respiratory depression, cardiotoxicity, seizures, tardive dyskinesias, extrapyradimal side-effects, neuroleptic malignant syndrome, sudden death and paradoxical deterioration of behaviour are also more likely at higher doses (Mackay, 1994).

Although controlled studies comparing standard doses with high doses of neuroleptics in treatment-resistant schizophrenia fail to show superior effectiveness of the 'megadose' regime (Kane, 1994), some patients do seem to respond to high doses of neuroleptics (Cookson. 1987) and a cautious trial of treatment may be justified. In such cases it would seem reasonable to expect that alternative management options have been considered (Tarrier, 1992), the patient's response to treatment and possible side-effects are carefully recorded and that the prescriber is a senior doctor. This becomes particularly important when medico-legal implications are considered.

In South Manchester Health District, there was no established system for identifying patients receiving doses of depot neuroleptics which exceed the maximum recommended by the British National Formulary (BNF).

\section{Aims}

(a) Identify the patients receiving depot neuroleptics at doses exceeding BNF maximum.

(b) Identify the reasons for using a high dose and whether any side-effects were recorded.

(c) Increase the awareness among consultant teams about these patients.

(d) Examine any changes in the prescribing habits of the consultant teams after they were made aware of such patients. 
Table 1. High-dose depot neuroleptics in south Manchester

\begin{tabular}{|c|c|c|c|c|c|c|}
\hline & \multicolumn{2}{|c|}{1994} & \multirow[b]{2}{*}{$\%$} & \multicolumn{3}{|c|}{1997} \\
\hline & Total & $>$ BNF dose & & Total & > BNF dose & $\%$ \\
\hline $\begin{array}{l}\text { Flupenthixol decanoate } \\
\text { Fluphenazine decanoate } \\
\text { Haloperidol decanoate } \\
\text { Pipothiazine palmitate } \\
\text { Zuclopenthixol decanoate } \\
\text { Fluspirilene }\end{array}$ & $\begin{array}{r}186 \\
57 \\
36 \\
34 \\
29 \\
1\end{array}$ & $\begin{array}{r}0 \\
15 \\
4 \\
1 \\
2 \\
0\end{array}$ & $\begin{array}{r}0 \\
26 \\
11 \\
3 \\
7 \\
0\end{array}$ & $\begin{array}{r}198 \\
57 \\
31 \\
40 \\
31 \\
0\end{array}$ & $\begin{array}{r}0 \\
11 \\
4 \\
3 \\
1 \\
0\end{array}$ & $\begin{array}{c}0 \\
19 \\
13 \\
7.5 \\
3 \\
0\end{array}$ \\
\hline
\end{tabular}

BNF, British National Formulary.

\section{The study}

Patients attending the psychiatric service of south Manchester receive depot injections from the depot clinic staff at Withington Hospital, community psychiatric nurses (CPNs), ward staff and hostel ward staff.

Between April and May 1994, all the patients receiving depot neuroleptics from the aforementioned services were screened and those patients receiving depots in excess of BNF maximums were identified.

A pro forma was developed to record demographic details; duration of illness; name of depot medication, dosage and duration; decision process for prescribing high dose; any assessment of efficacy and possible side-effects.

The results of this first phase of the audit were presented at a departmental audit meeting. Individual consultants were then sent information about the $\mathrm{BNF}$ maximum recommendations and a list of their patients who were receiving depots above this maximum.

Between April and May 1997, three years after the first audit and over two years after the consultants were made aware of the results, the audit was repeated using the same process of identification and pro forma.

\section{Findings}

In 1994,343 patients received depot neuroleptics from the psychiatric services of south Manchester: 235 from the depot clinic and ward staff; 97 from CPNs; and 11 from hostel ward staff. In 1997, 357 patients received depot neuroleptics: 219 from the depot clinic and ward staff; 134 from CPNs; and four from hostel ward staff.

In 1994, 22 patients (6\%) received depot medication in doses exceeding the BNF maximum, compared with $19(5 \%)$ in 1997. There were no significant differences between the groups in 1994 and 1997 with respect to gender. age, diagnosis, marital status, employment and living situation. The mean duration of illness was
14 years in 1994 and 20 years in 1997. Patients were prescribed medication above the BNF maximum dose for 30.55 months and $\mathbf{5 4 . 5 3}$ months in 1994 and 1997 respectively.

Just over half the prescriptions for depot medication were for flupenthixol decanoate, but none was above the BNF maximum (Table 1). In 1994 and 1997 the proportions of prescriptions of fluphenazine decanoate (26 and 19\%), haloperidol decanoate (11 and $13 \%)$, pipothiazine palmitate (3 and $7.5 \%)$ and zuclopenthixol decanoate (7 and $3 \%$ ) were above the upper limit of the BNF respectively.

During both phases of the audit, in the majority of cases there was no formal record of the dose being above the BNF maximum. The reason for increasing the dose above BNF maximum was documented in most of the cases. The most common reason was persistent psychotic symptoms in 77 and $67 \%$ of cases in 1994 and 1997 respectively. Other reasons included a desire to convert oral and depot medication to depot alone and as sedation in one patient with agitation and insomnia.

In both phases of the audit, the decision to increase the dose was made by a senior doctor (consultant or senior registrar) in the majority of cases $(58 \%)$. In $27 \%$ of cases there was no record of a discussion with the consultant when the decision was made by a junior doctor (registrar or senior house officer). For those patients whose depots were increased above the BNF upper limit by a senior doctor in 1994 , there was a trend for the dose to be reduced in 1997 (Fisher's exact test, two-tailed, $P=0.1$ ). Twenty-seven per cent of patients were not reviewed by the consultants either prior to, or after the dose was increased above the BNF maximum.

Oral antipsychotic medications were prescribed in 50 and $68 \%$ of patients in 1994 and 1997 respectively. These included chlorpromazine, thioridazine, droperidol and trifluoperazine. When all the subjects in 1994 and 1997 were included, side-effects were recorded in $36 \%$ of subjects. Extrapyradimal side-effects were 
recorded in $31.8 \%$ and $31.6 \%$ of patients in 1994 and 1997 respectively. One patient in 1994 and two in 1997 were noted to have tardive dyskinesia.

The consultant team that conducted this audit demonstrated a change in prescribing. There were seven patients receiving medication above BNF maximum dose in 1994. Of the seven patients only one was still prescribed a depot dose above the BNF maximum in 1997. The reduction in the proportion of patients from 32 $(7 / 22)$ to $5 \%(1 / 19)$ was significant (Fisher's exact two-tailed $P<0.05$ ).

The remaining seven consultant teams had 15 patients taking medication above BNF maximum in 1994 and 18 in 1997 . Ten of the 18 patients in 1997 were patients who were identified in the 1994 cohort, four new patients had their doses increased above BNF maximum and the remaining four were transferred from the long-stay wards at a nearby large mental hospital on the doses above BNF maximum.

Of the 12 patients from 1994 who were not identified in 1997 audit, one was transferred to another hospital and lost to follow-up. Of the remaining 11 patients, two were receiving a reduced dose of the same depot antipsychotic, one patient was taking flupenthixol decanoate and another was given zuclopenthixol decanoate. All the doses were below the BNF maximum. Seven patients were receiving atypical antipsychotics: clozapine $(n=3)$, risperidone $(n=3)$ and sertindole $(n=1)$.

\section{Comment}

This audit was based on a retrospective review of case notes and was dependent on the accuracy of information recorded in the notes by medical staff. It only examined patients who received depots above the BNF maximum. It did not review patients who were receiving depot medication below the BNF maximum, but who were also receiving high doses of oral antipsychotic medication. Thus, the proportion of patients receiving 'high-dose neuroleptics' in this study may be an underestimate of the total population at risk. The results from this audit may not be generalised to other districts as the patients and the therapeutic strategies may not be representative of other districts in the UK.

In contrast to a similar audit performed in Horton Hospital (Warner et al, 1995), there was little overall reduction in the number of patients receiving depots above the BNF maximum. However, half of the patients identified in 1994 were receiving either depot antipsychotic medication below the BNF maximum dose or atypical antipsychotic medication at the time of follow-up in 1997. Taylor \& Cookson (1997), in another audit of high-dose medication, found that the reduction in the dose of depot medication varied, with $40 \%$ of patients receiving decreased doses and $30 \%$ increased doses over a two-year period.

Although flupenthixol decanoate was the most commonly used depot medication, fluphenazine decanoate was the depot most frequently prescribed above the BNF maximum. Part of the explanation for this skewed distribution may be attributable to marketing. However, flupenthixol decanoate has much higher BNF maximum dose limit in chlorpromazine equivalence, that is, $2000 \mathrm{mg}$ per day compared with other depot antipsychotic medication $300-600 \mathrm{mg}$ per day (Foster, 1989).

Significant changes in the prescribing habits of the consultant team that undertook this audit were evident, but the effect did not generalise to other consultant teams. To increase awareness among colleagues, the depot prescription cards will have the $\mathrm{BNF}$ maximum recommendations written in bold at the top, and those exceeding these limits will be highlighted. Individual letters on each patient will be sent to the responsible medical officer together with copies of the Royal College of Psychiatrists' guidelines on the use of high-dose antipsychotic medication (Thompson. 1994). Different consultant teams will be encouraged to take the responsibility of conducting similar audits in the future.

\section{References}

Cookson. I. B. (1987) The effect of a $50 \%$ reduction of $\mathrm{Cis}(\mathrm{z})$ flupenthixol decanoate in chronic schizophrenic patients maintained on high dose regime. International Clinical Psychopharmacology. 2. 141-149.

FOSTER. P. (1989) Neuroleptic equivalence. Pharmaceutical Journal, 243, 431-432.

HiRSCH. S. R. \& BARNES. T. R. E. (1994) Clinical use of highdose neuroleptics. British Journal of Psychiatry. 164. 94-96.

KANE, J. M. (1994) The use of higher-dose antipsychotic medication. British Journal of Psychiatry. 164. 431-432.

KING. D. (1994) The use of high doses of neuroleptics: the current situation. International Clinical Psychopharmacology, 9, 75-78.

MACKAY, A. V. P. (1994) High-dose antipsychotic medication. Advances in Psychiatric Treatment. 1. 16-22.

TARRIER. N. (1992) Management and modification of residual positive psychotic symptoms. In Innovations in the Psychological Management of Schizophrenia (eds M. Birchwood \& N. Tarrier). Chichester: John Wiley.

TAYLOR. J. R. \& CoOKSON, I. B. (1997) Audit of out-patients on 'higher dose' antipsychotics. Psychiatric Bulletin. 21. 445-458.

THOMPSON. C. (1994) The use of high-dose antipsychotic medication. British Journal of Psychiatry. 164. 448-458.

WARNER, J. P., Slade, R. \& BARNes. T. R. E. (1995) Change in neuroleptic prescribing practice. Psychiatric Bulletin. 19. 237-239. 
N. Purandare, Senior Registrar in Psychiatry, University Hospital of South Manchester; L. Aitken, Consultant in Old Age Psychiatry, Stepping Hill Hospital, Stockport; P. Joshi, Consultant Psychiatrist, Stepping Hill Hospital; and ${ }^{*} \mathrm{C}$. S. Thomas, Consultant Psychiatrist,
Department of Psychiatry. University Hospital of South Manchester, Manchester M20 8LR

\title{
Enforcing treatment with clozapine: survey of views and practice
}

\author{
Stephen Pereira, Dominic Beer and Carol Paton
}

\begin{abstract}
Aim and method The aim of the study was to survey strategies for dealing with patients who refuse clozapine blood tests or tablets. One hundred and twenty-five psychiatric intensive care unit consultants were sent a questionnaire.

Results Thirty-nine (31\%) questionnaires were returned. Opinions and practices were diverse ranging from uncertainty around the legal right to enforce venepuncture, to the practice of slipping clozapine into a patient's drink without their knowledge.

Clinical implications There is need for an open debate of the relevant legal and ethical issues.
\end{abstract}

Neuroleptic treatment resistance in schizophrenia is a well acknowledged and not uncommon phenomenon (Morrison, 1996). Some severely ill people may be a danger towards others or themselves, or be at risk from severe self-neglect. These features arise directly from the person's illness. Clozapine is the only antipsychotic proven to be effective in people who have treatment-resistant schizophrenia (Kane, 1992). As well as having positive effects on aggression (Special Hospitals Treatment Resistant Schizophrenia Research Group, 1996) and suicidal behaviour (Meltzer \& Okayli, 1995), clozapine is associated with a very low incidence of extrapyramidal side-effects (Kane, 1992), which contributes towards its improved efficacy against negative symptoms. Although some subjects respond markedly to clozapine in the first few weeks of treatment, for others response may be slower and assessment periods of up to one year have been advocated (Kane, 1992). The tangible benefits that clozapine offers cannot be realised when subjects refuse treatment due to lack of insight or fear of blood tests.

Many psychiatric intensive care units (PICUs), challenging behaviour and forensic units have a small core group of patients who are referred to them out of despair; this may not only be due to acutely or chronically disturbed mental state and behaviour, but also due to the fact that every other intervention (e.g. maximum or above maximum British National Formulary doses of oral or depot neuroleptics, neuroleptic combinations, adjunct medications and psychological interventions) has been tried with limited or no success. In the meantime, the individual continues to behave dangerously towards others (e.g. destruction of property, assaults, arson, sexual disinhibition) or towards themselves (e.g. repetitive self-mutilation, severe self-neglect). Such ongoing behaviour poses the question of 'duty of care', not only to the patient themselves, but also to others at risk from these behaviours. In this small minority of patients who cannot be contained safely on open wards or sometimes even on PICUs, and who refuse to cooperate with clozapine treatment, the issue of enforcing blood tests and then subsequently, oral clozapine, may arise as one of the treatment options. As such interventions are more likely to be carried out on PICUs than on open admission wards, we sought the opinions of PICU consultants on the legal, ethical and practical considerations that may underlie such a treatment intervention and sought to determine if any units had practical experience of enforcing treatment with clozapine. 